\title{
A generic hidden Markov model for multi-parent populations
}

\author{
Karl W. Broman*,1
}

*Department of Biostatistics and Medical Informatics, University of Wisconsin-Madison,

Madison, Wisconsin 53706

2021-11-01 
Running head: Generic HMM for MPPs

Key words: quantitative trait loci, QTL, HMM, Collaborative Cross, Diversity Outbred mice, heterogeneous stock, MPP, multiparental populations, Multiparent Advanced Generation Inter-Cross (MAGIC)

\title{
${ }^{1}$ Corresponding author:
}

\author{
Karl W Broman
}

Department of Biostatistics and Medical Informatics

University of Wisconsin-Madison

2126 Genetics-Biotechnology Center

425 Henry Mall

Madison, WI 53706

Phone: 608-262-4633

Email: broman@wisc.edu 


\section{Abstract}

A common step in the analysis of multi-parent populations is genotype reconstruction: identifying the founder origin of haplotypes from dense marker data. This process often makes use of a probability model for the pattern of founder alleles along chromosomes, including the relative frequency of founder alleles and the probability of exchanges among them, which depend on a model for meiotic recombination and on the mating design for the population. While the precise experimental design used to generate the population may be used to derive a precise characterization of the model for exchanges among founder alleles, this can be tedious, particularly given the great variety of experimental designs that have been proposed. We describe an approximate model that can be applied for a variety of multi-parent populations. We have implemented the approach in the R/qt12 software, and we illustrate its use in applications to publicly-available data on Diversity Outbred and Collaborative Cross mice. 


\section{Introduction}

Multi-parent populations (MPPs) are valuable resources for the analysis of complex traits (de Koning and McIntyre 2017), including the mapping of quantitative trait loci (QTL). A wide variety of MPPs have been developed, including heterogeneous stock (HS) in mice (Mott et al. 2000) and rats (Solberg Woods et al. 2010), eight-way recombinant inbred lines (RIL) in mice (Complex Trait Consortium 2004) and Drosophila (King et al. 2012), and multi-parent advanced generation intercross (MAGIC) populations in a variety of plant species including arabidopsis (Kover et al. 2009), wheat (Cavanagh et al. 2008), maize (Dell'Acqua et al. 2015), and rice (Bandillo et al. 2013).

QTL mapping in MPPs can be performed through statistical tests at individual single nucleotide polymorphisms (SNPs), as used in genome-wide association studies. However, many investigators first seek to reconstruct the mosaic of founder haplotypes along the chromosomes of MPP individuals and use this reconstruction to test for association between founder alleles and the quantitative phenotype. This approach was first introduced by Mott et al. (2000) for the analysis of HS mice, implemented in the HAPPY software, and has been continued in packages such as R/mpMap (Huang and George 2011), DOQTL (Gatti et al. 2014), and R/qtl2 (Broman et al. 2019a).

The process of genotype reconstruction in an MPP individual is illustrated in Fig. 1. The genotypes in the founder strains (Fig. 1a) and the MPP offspring (Fig. 1b) are used to calculate the probability of each possible founder genotype at each position along the chromosome (Fig. 1c). Thresholding of these probabilities can be used to infer the founder genotypes and the locations of recombination breakpoints (Fig. 1d). 
a

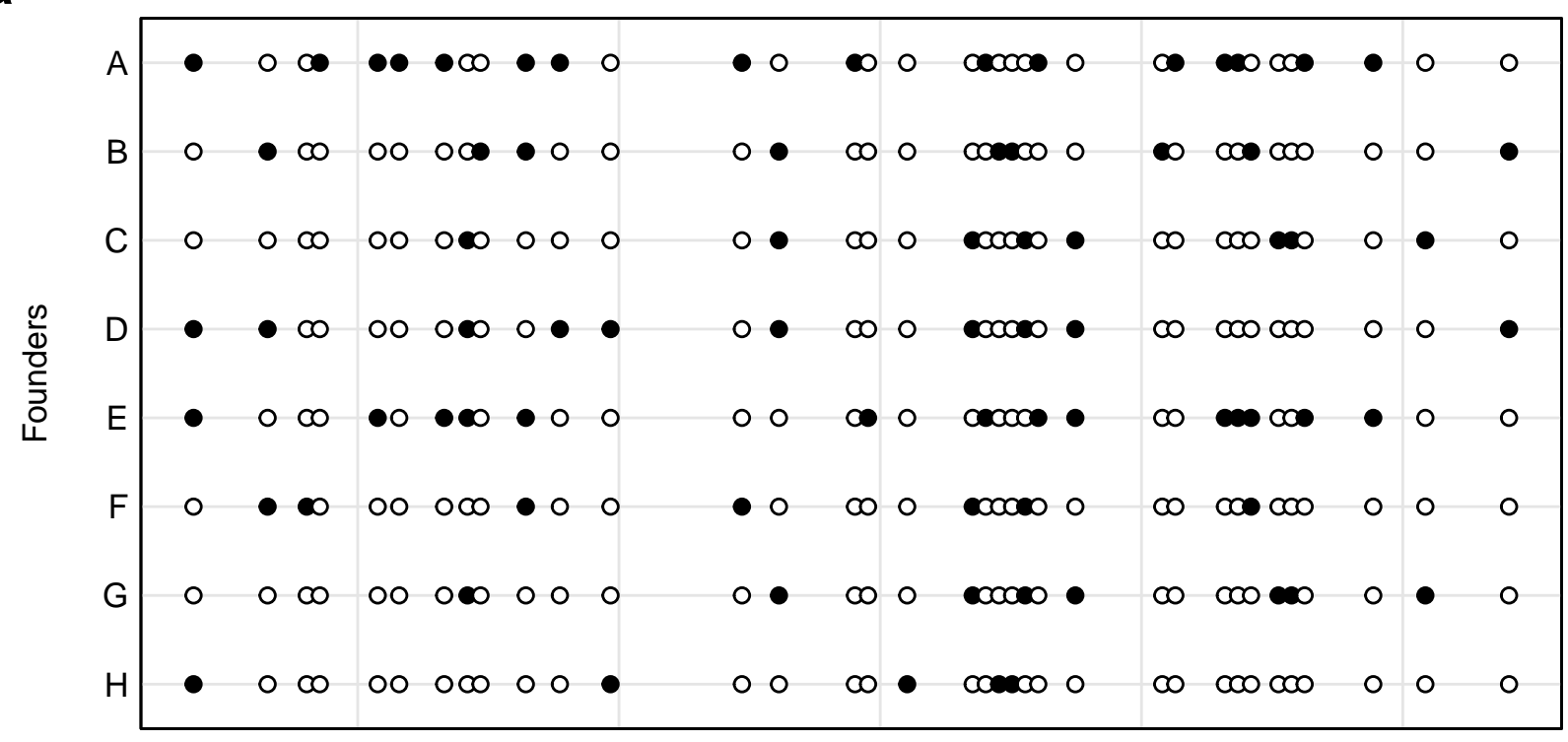

b

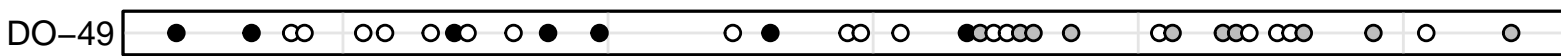

C

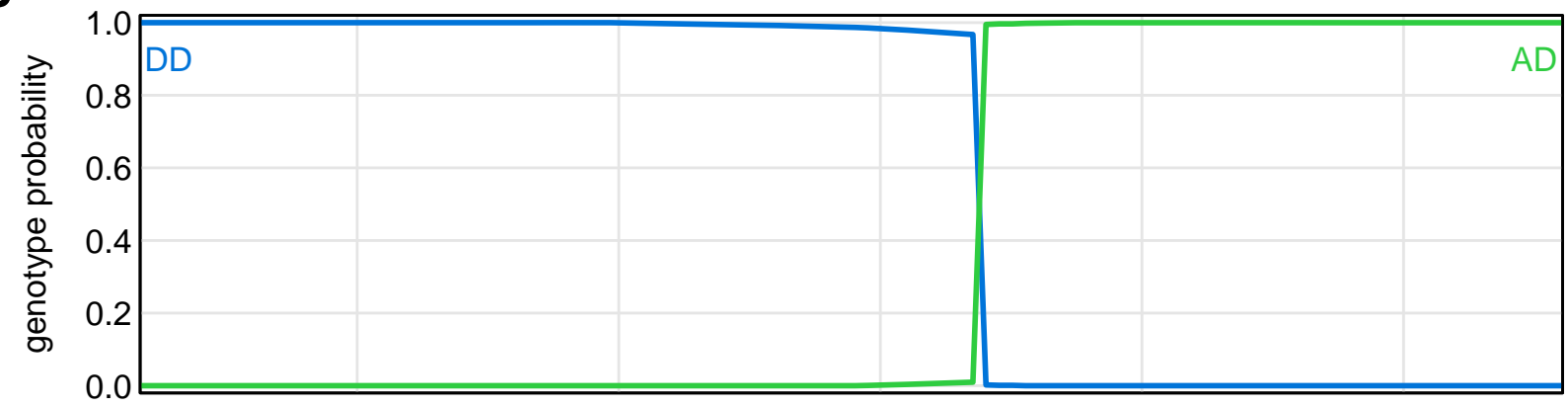

d

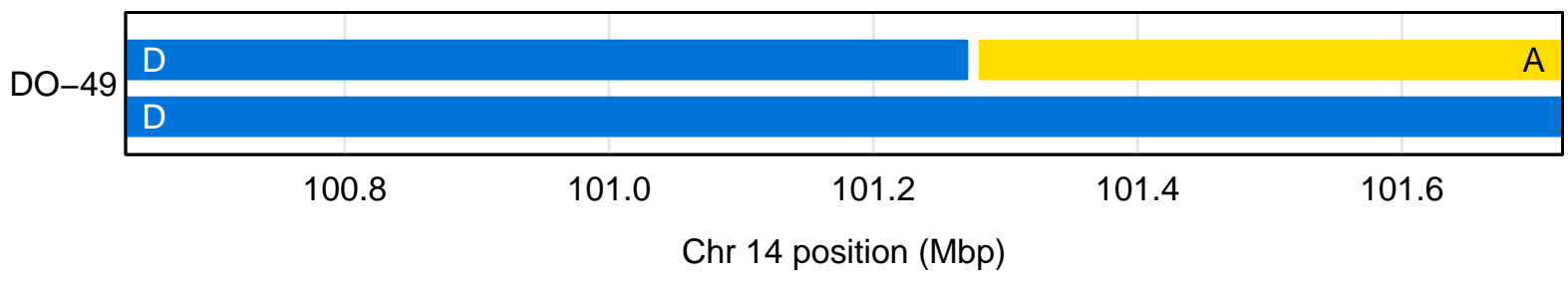

Figure 1 Illustration of genotype reconstruction in a $1 \mathrm{Mbp}$ region in a single Diversity Outbred (DO) mouse. a. Genotypes of eight founder strains at a set of SNPs, with open and closed circles corresponding to being homozygous for the more-frequent and less-frequent allele, respectively. b. Genotype of the DO mouse at the SNPs, with gray indicating the mouse is heterozygous. c. Genotype probabilities for the DO mouse along the chromosome segment, given the observed data. Genotypes other than the two shown have negligible probability across the region. d. Inferred haplotypes in the DO mouse. 
Such genotype reconstructions are valuable not just for QTL analysis but also for data diagnostics (Broman et al. 2019b). For example, the inferred number of recombination breakpoints is a useful diagnostic for sample quality. Further, the reconstructed genotypes can be used to derive predicted SNP genotypes; comparing these to the observed SNP genotypes can help to identify problems in both samples and SNPs.

The probability calculation in Fig. 1c depends on a model for the process along MPP chromosomes in Fig. 1d. In the HAPPY software for HS mice, Mott et al. (2000) used a model of random mating in a large population. Broman (2005) extended the work of Haldane and Waddington (1931) to derive two-locus genotype probabilities in multi-parent recombinant inbred lines. This was later developed for the case of multi-parent advanced intercross populations (Broman 2012a,b), including Diversity Outbred (DO) mice (Churchill et al. 2012).

Genotype reconstruction for a variety of MPP designs has been implemented in the R/qt12 software (Broman et al. 2019a, https: / / kbroman.org/qt 12). But it can be tedious analytical work to derive the appropriate transition probabilities for each new MPP design that is proposed. An alternative is to develop a more general approach for genotype reconstruction, such as used in the software RABBIT (Zheng et al. 2015). However, this approach has a variety of parameters that can be difficult to specify.

Here we propose a similarly general method for genotype reconstruction in MPPs. We imagine that an MPP was derived from a population of homozygous founder strains at known proportions, $\alpha_{i}$, followed by $n$ generations of random mating among a large number of mating pairs. We can derive the exact transition probabilities for this situation. The $\alpha_{i}$ should be simple to specify from the MPP design, and the effective number of generations of random mating, $n$, can be determined by computer simulation, to match the expected density of recombination 
breakpoints.

Our approach has been implemented in R/qt12. While we currently focus on data with SNP genotype calls, such as from microarrays, our model could potentially be incorporated into methods for genotype imputation from low-coverage sequencing, such as that of Zheng et al. (2018). We illustrate our approach through application to publicly-available datasets on DO (Al-Barghouthi et al. 2021) and Collaborative Cross mice (Srivastava et al. 2017). 


\section{Methods}

For genotype reconstruction in a multi-parent population (MPP), we use a hidden Markov model (HMM; see Rabiner 1989). Our basic approach is as described in Broman and Sen (2009, App. D) for a biparental cross; the extension to an MPP is straightforward and described below.

Consider an MPP derived from $k$ inbred lines. We focus on a single individual, and on a single chromosome with $M$ marker positions (including pseudomarkers: positions between markers at which we have no data but would like to infer the underlying genotype). Let $G_{m}$ be the underlying genotype at position $m$. In a homozygous population, such as RIL, the $G_{m}$ take one of $k$ possible values, the $k$ homozygous genotypes. In a heterozygous population, such as advanced intercross lines (AIL), the $G_{m}$ take one of $\left(\begin{array}{l}k \\ 2\end{array}\right)+k$ possible values, the $\left(\begin{array}{l}k \\ 2\end{array}\right)$ heterozygotes and $k$ homozygotes. Let $O_{m}$ be the observed SNP genotype at position $m$ (possibly missing). We assume that the $G_{m}$ form a Markov chain (that $G_{1}, \ldots, G_{m-1}$ are conditionally independent of $G_{m+1}, \ldots, G_{M}$, given $G_{m}$ ), and that $O_{m}$ is conditionally independent of everything else, given $G_{m}$. The forward-backward algorithm (see Rabiner 1989) takes advantage of the conditional independence structure of the HMM to calculate $\operatorname{Pr}\left(G_{m} \mid \boldsymbol{O}\right)$.

The key parameters in the model are the initial probabilities, $\pi_{g}=\operatorname{Pr}\left(G_{1}=g\right)$, the transition probabilities, $t_{m}\left(g, g^{\prime}\right)=\operatorname{Pr}\left(G_{m+1}=g^{\prime} \mid G_{m}=g\right)$, and the emission probabilities, $e_{m}(g)=\operatorname{Pr}\left(O_{m} \mid G_{m}=g\right)$. A particular advantage of the HMM for genotype reconstruction is the easy incorporation of a model for genotyping errors (Lincoln and Lander 1992), which is done through the emission probabilities, which condition on the founder SNP genotypes but allow some fixed probability $\epsilon$ that the observed SNP genotype in the MPP individual is in error and incompatible with the underlying genotype $G_{m}$ and the SNP genotypes in the founder lines. 
The initial and transition probabilities govern the underlying Markov chain, including the relative frequency of founder alleles and the frequency of recombination breakpoints along MPP chromosomes. In principle, these probabilities may be derived on the basis of the crossing design for the MPP. In practice, the transition probabilities can be tedious to derive, and exact calculations may provide no real advantage for genotype reconstruction.

Here, we derive the transition probabilities for a generic MPP design, which may then be applied generally. We consider a founder population with $k$ inbred lines in proportions $\alpha_{i}$, and imagine subsequent generations are produced by random mating with a very large set of mating pairs.

Consider a pair of loci separated by a recombination fraction of $r$ (assumed the same in both sexes) and let $p_{i j}^{(n)}$ be the probability of that a random haplotype at generation $n$ has alleles $i$ and $j$. At $n=0$, we have just the founding inbred lines, and so $p_{i j}^{(0)}=\alpha_{i}$ if $i=j$ and $=0$ if $i \neq j$.

The probabilities from one generation to the next are related by a simple recursion, as in Broman (2012b). Consider a random haplotype at generation $n$. It was either a random haplotype from generation $n-1$ transmitted intact without recombination, or it is a recombinant haplotype bringing together two random alleles. Thus

$$
p_{i j}^{(n)}=(1-r) p_{i j}^{(n-1)}+r \alpha_{i} \alpha_{j}
$$

Using the same techniques described in Broman (2012b), we find the solutions:

$$
p_{i j}^{(n)}= \begin{cases}\alpha_{i}^{2}+(1-r)^{n} \alpha_{i}\left(1-\alpha_{i}\right) & \text { if } i=j \\ \alpha_{i} \alpha_{j}\left[1-(1-r)^{n}\right] & \text { if } i \neq j\end{cases}
$$


The transition probabilities along a haplotype are derived by dividing the above by the marginal probability, $\alpha_{i}$. Thus if $G_{1}$ and $G_{2}$ are the genotypes at the two loci, we have the following transition probabilities.

$$
\operatorname{Pr}\left(G_{2}=j \mid G_{1}=i\right)= \begin{cases}\alpha_{i}+(1-r)^{n}\left(1-\alpha_{i}\right) & \text { if } i=j \\ \alpha_{j}\left[1-(1-r)^{n}\right] & \text { if } i \neq j\end{cases}
$$

For a heterozygous population (such as heterogeneous stock or Diversity Outbred mice), an individual will have two random such haplotypes. For homozygous population (such as MAGIC), we treat them like doubled haploids, by taking a single random chromosome and doubling it.

For the $\mathrm{X}$ chromosome, we use the same equations but replace $n$ with $(2 / 3) n$, since recombination occurs only in females, so in $2 / 3$ of the $X$ chromosomes. This provides a remarkably tight approximation.

You can potentially use the expected number of crossovers to calibrate the number generations of random mating, or the map expansion, which is the relative increase in the number of crossovers. Let $R(r)$ be the chance that a random haplotype has an exchange of alleles across an interval with recombination fraction $r$, so $R(r)=1-\sum_{i} p_{i i}^{(n)}$. The map expansion is $d R / d r$ evaluated at $r=0$ (see Teuscher and Broman 2007). Using equation (2) above, we then get that the map expansion in this population is $n\left(1-\sum \alpha_{i}^{2}\right)$. In the special case that $\alpha_{i} \equiv 1 / k$ for all $i$, this reduces to $n(k-1) / k$.

The map expansion at generation $s$ in DO mice on an autosome is $(7 / 8)(s-1)+M_{1}$ where $M_{1}$ is the weighted average of map expansion in the pre-CC founders (Broman 2012b), or about $(7 s+37) / 8$. Equating this with $(7 / 8) n$, we can thus take $n \approx s+5$ when using this model to 
bioRxiv preprint doi: https://doi.org/10.1101/2021.08.03.454963; this version posted November 1, 2021. The copyright holder for this preprint

(which was not certified by peer review) is the author/funder, who has granted bioRxiv a license to display the preprint in perpetuity. It is made available under aCC-BY 4.0 International license.

approximate the DO. For the Collaborative Cross, Broman (2005) showed that $\mathrm{R}=7 \mathrm{r} /(1+6 \mathrm{r})$, and so the map expansion is 7 . Thus we can take $n=8$ as the effective number of generations of random mating. 


\section{Applications}

We illustrate our approach with application to datasets on Diversity Outbred mice (Al-Barghouthi et al. 2021) and Collaborative Cross (CC) mice (Srivastava et al. 2017). In both cases, the approach provided results that were generally equivalent to those from the more exact model, though with important differences in the results for the $\mathrm{X}$ chromosome in the $\mathrm{CC}$ application.

\section{Diversity Outbred mice}

The Diversity Outbred mouse data of Al-Barghouthi et al. (2021) concerns a set of 619 mice from DO generations 23-33, in 11 batches by generation and including 304 females and 315 males. The mice were genotyped on the GigaMUGA array (Morgan et al. 2016) and the cleaned data consist of genotypes at 109,427 markers. A wide variety of phenotypes are available; we focus on the 20 contributing to the results in Table 1 of Al-Barghouthi et al. (2021).

We performed genotype reconstruction using the transition matrices derived specifically for DO mice (Broman et al. 2019b) as well as by the approximate model proposed above. For the DO mice at generation $n$, we used the transition probabilities for general 8-way advanced intercross lines (AIL) at $n+5$.

Following Al-Barghouthi et al. (2021), we assumed a 0.2\% genotyping error rate and used the Carter-Falconer map function (Carter and Falconer 1951). Calculations were performed in R ( $R$ Core Team 2021) with R/qtl2 (Broman et al. 2019a), on an 8-core Linux laptop with 64 GB RAM. The calculations with the DO-specific model took approximately $35 \mathrm{~min}$, while those with the general AIL model took 27 min, an almost $25 \%$ reduction in computation time. 
a

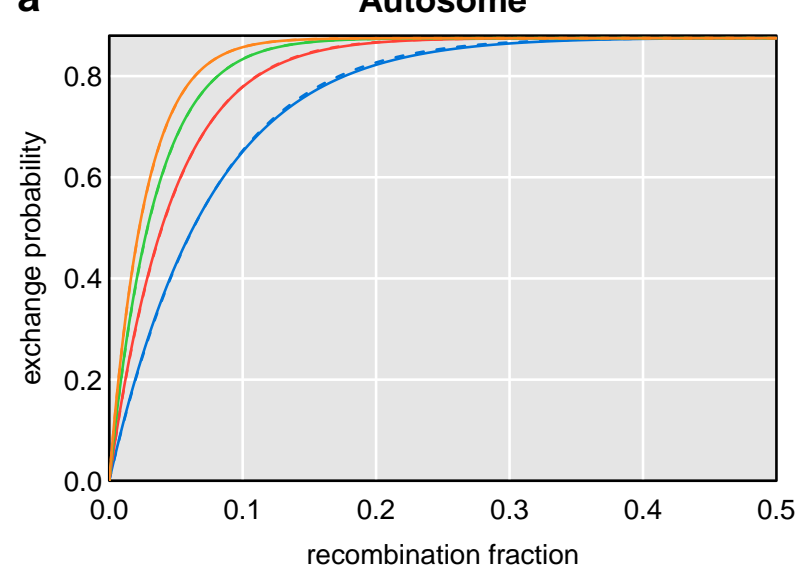

b

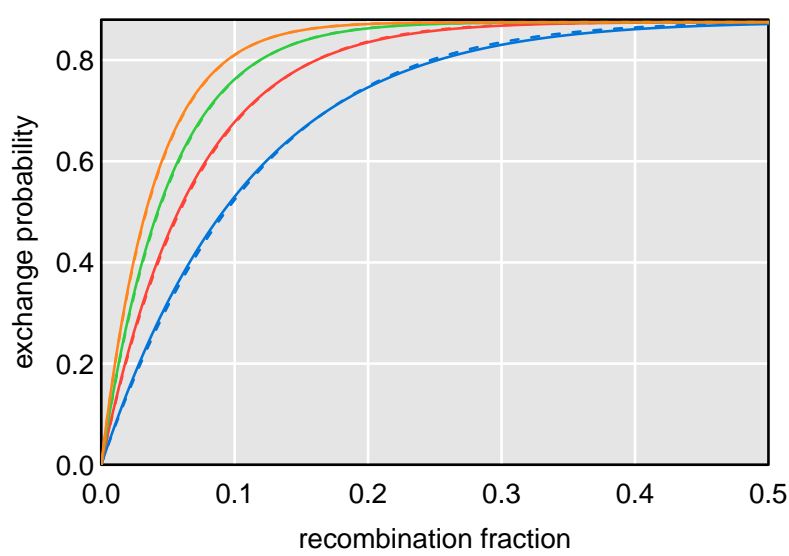

C

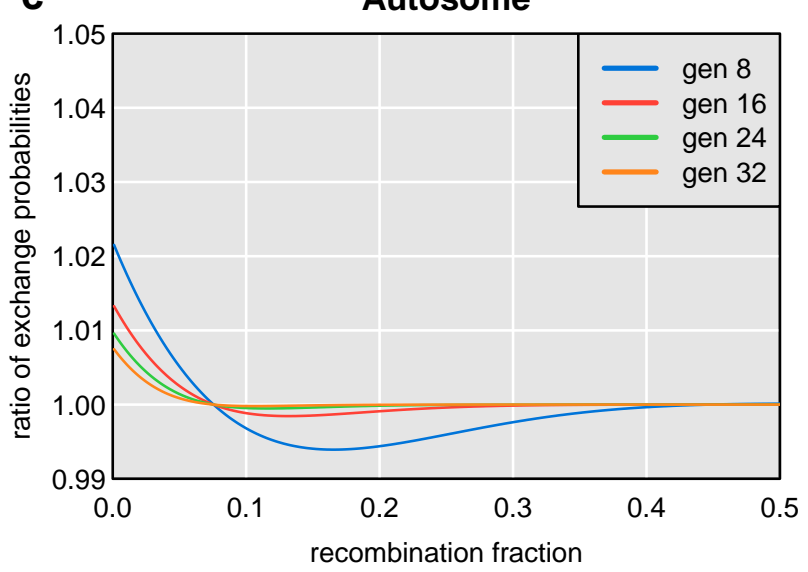

d

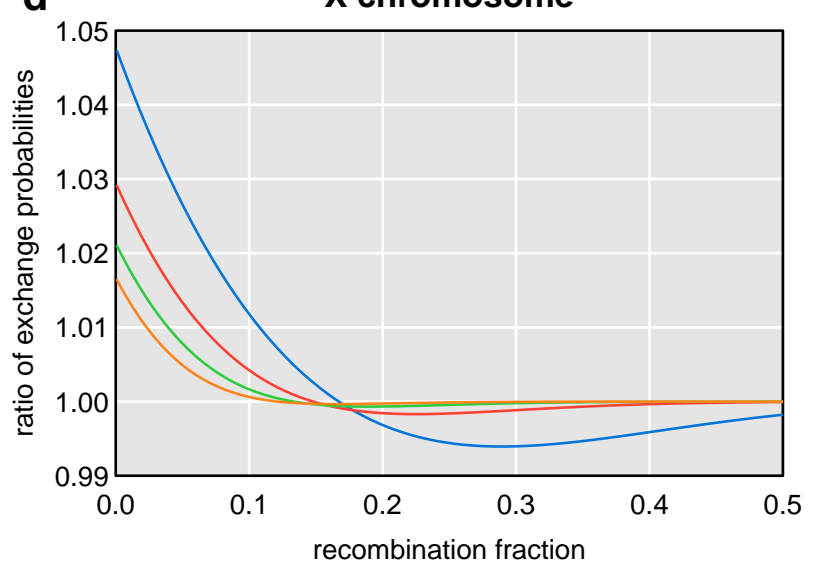

Figure 2 Differences in transition probabilities for Diversity Outbred mice from more-exact calculations and the proposed approximations. Probability of an exchange of alleles across an interval as a function of generation with the more-exact calculations (solid lines) and the proposed approximation (dashed lines) for autosomes (a) and the X chromosome (b). Ratio of the probabilities (more-exact versus approximation) for autosomes (c) and the $\mathrm{X}$ chromosome (d).

The transition probabilities used by the two models are only subtly different and become less different in later generations. The probability of an exchange across an interval on a random DO chromosome, as a function of the recombination fraction for the interval and the number of generations, is shown in Fig. 2.

QTL analysis proceeded by the method described in Gatti et al. (2014) and also used by Al-Barghouthi et al. (2021). Namely, we fit a linear mixed model assuming an additive model for the founder haplotypes, with a residual polygenic effect to account for relationships among 
individuals with kinship matrices calculated using the "leave-one-chromosome-out" (LOCO) method (see Yang et al. 2014), and with a set of fixed-effect covariates defined in Al-Barghouthi et al. (2021).

The genotype probabilities were almost indistinguishable. The maximum difference was 0.011 on the $\mathrm{X}$ chromosome followed by a difference of 0.007 on chromosome 8 . For that reason, the QTL mapping results were hardly different. Across all 20 traits considered, the maximum difference in LOD scores in the two sets of results was 0.02 .

The LOD curves by the two methods for tissue mineral density (TMD) and the differences between them are shown in Fig. 3. The QTL on chromosomes 1 and 10 have LOD scores of 23.9 and 14.6, respectively, but the maximum difference in LOD, genome-wide, between the two methods is just 0.014 .

\section{Collaborative Cross mice}

As a second application of our approach, we consider the data for a set of 69 Collaborative Cross (CC) lines (Srivastava et al. 2017). These are eight-way recombinant inbred lines (RIL) derived from the same eight founders as the DO mice, as the DO was formed from 144 partially-inbred lines from the process of developing the CC (Svenson et al. 2012).

Each CC line was formed from a separate "funnel," bringing the eight founder genomes together as rapidly as possible, for example $[(\mathrm{A} \times \mathrm{B}) \times(\mathrm{C} \times \mathrm{D})] \times[(\mathrm{E} \times \mathrm{F}) \times(\mathrm{G} \times \mathrm{H})]$, where the female parent is listed first in each cross. Inbreeding was accomplished by repeated mating between siblings.

The recombination probabilities for the autosomes in the $\mathrm{CC}$ do not depend on the order of the 
a
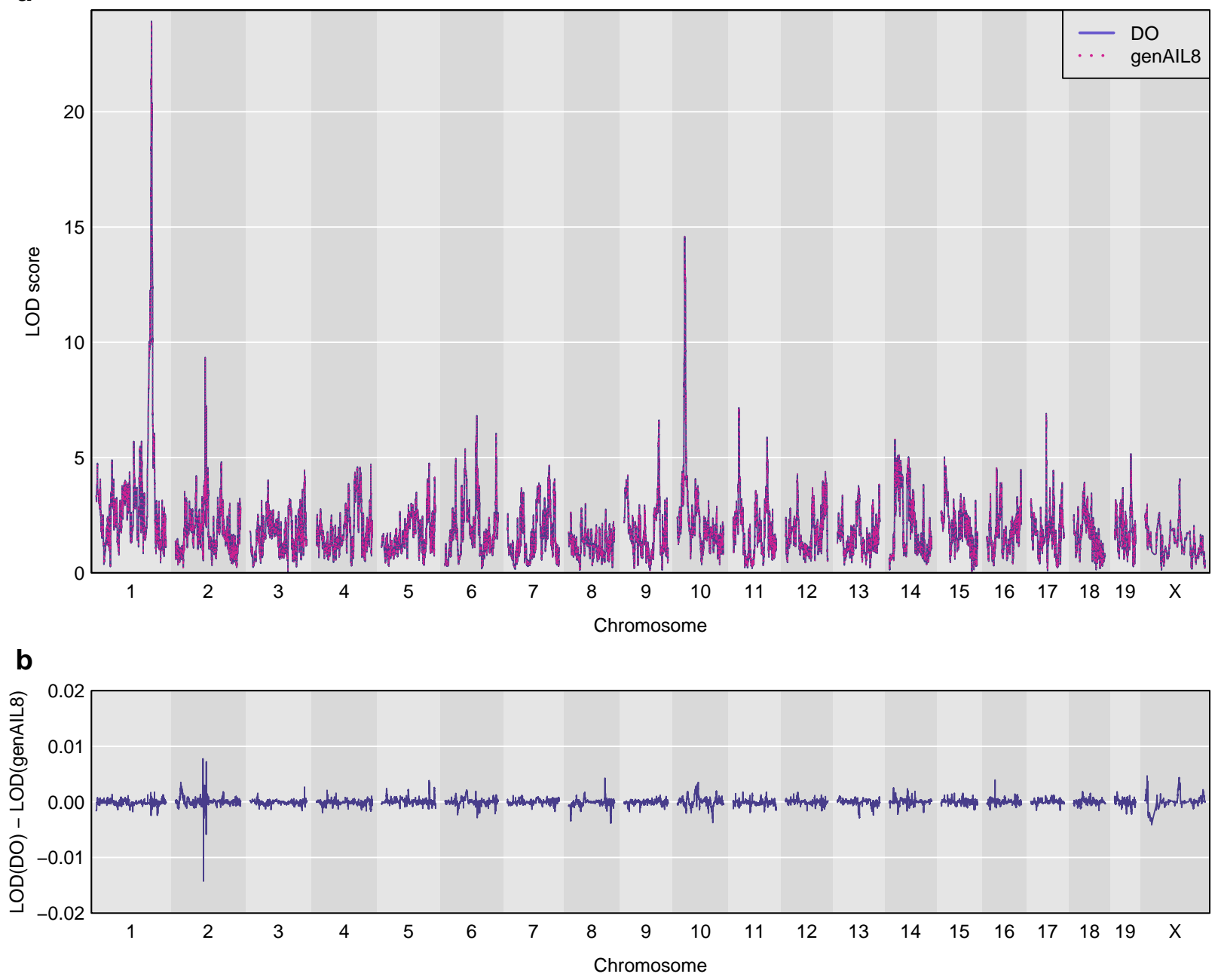

Figure 3 Genome scan for tissue mineral density (TMD) for the DO mouse data from AlBarghouthi et al. (2021). a. LOD curves across the genome using the genotype probabilities from the DO-specific model (solid blue curves) and the proposed general model (dotted pink curves). $\mathbf{b}$. Differences between the two sets of LOD curves. 
founders in the funnel for a line (Broman 2005). This is in contrast with the case of 8-way RIL by selfing (see Broman 2005, Table 2). For the X chromosome, however, the cross order is important, as only 5 of the 8 founders can contribute. For example, in a line derived from the cross $[(A \times B) \times(C \times D)] \times[(E \times F) \times(G \times H)]$, the single-locus genotype probabilities on the $X$ chromosome are 1/6 each for alleles A, B, E, and F, and 1/3 for allele C, while alleles D, G, and H will be absent. And note that the mitochondrial DNA will come from founder A, while the Y chromosome will be from founder $\mathrm{H}$.

The cross funnel information was missing for 14 of the $69 \mathrm{CC}$ lines. While the sources of the mitochondria and Y chromosome were provided for all lines, there were several inconsistencies in these data: line CC013/GeniUnc has the same founder listed as the source for its mitochondria and Y chromosome, and for three lines (CC031/GeniUnc, CC037/TauUnc, and CC056/GeniUnc) the founder on the $\mathrm{Y}$ chromosome is also seen contributing to the $\mathrm{X}$ chromosome. We used the genotype probabilities reported in Srivastava et al. (2017) to construct compatible cross funnels, with small modifications to handle the inconsistent information.

We performed genotype reconstruction using the transition matrices derived specifically for CC mice (Broman 2005) as well as by the approximate model proposed above, using $n=8$ generations of random mating, chosen to match the expected frequency of recombination breakpoints.

The resulting probabilities were nearly identical on all autosomes in all CC lines. The maximum difference in probabilities on the autosomes was just 0.0006 .

There were some important differences on the $\mathrm{X}$ chromosome, however. There were no cases with high probability pointing to different founder alleles by the two models, but there were several cases where two or more founders cannot be distinguished, but some would be excluded 
by the assumed cross design.

For example, in Figure 4, we show the genotype probabilities along the $\mathrm{X}$ chromosome for strain CC038/GeniUnc, as calculated with the more-exact model (Figure 4a) and with the approximate model (Figure 4b). We also include the results for the case that the more-exact model but when an incorrect cross design was used (Figure 4c). Note the segment near $135 \mathrm{Mbp}$, which is inferred to be from founder NOD with the more-exact model but is equally likely B6 or NOD with the approximate model; the B6 and NOD founder strains are identical in the region, but the assumed cross design for the CC038/GeniUnc strain excluded B6. For the results using the incorrect cross design (which excluded not just B6 but also 129 and NOD), the results across the entire chromosome become a chopped-up mess, with an apparent 39 recombination breakpoints, versus 5 when the correct cross information is used.

Overall, there were seven strains where the maximum difference in the probabilities from the more-exact model and the proposed approximate model were in the range $0.25-0.50$, and another eight strains with maximum difference in the range $0.10-0.25$. All of the differences concern cases where multiple founders are identical for a region and either some would be excluded by the cross design, or where the difference in prior frequencies affects the results. For example, in the cross $[(\mathrm{A} \times \mathrm{B}) \times(\mathrm{C} \times \mathrm{D})] \times[(\mathrm{E} \times \mathrm{F}) \times(\mathrm{G} \times \mathrm{H})]$, the frequency of the $\mathrm{C}$ allele on the $\mathrm{X}$ chromosome is twice that of A, B, E, and F. 
a

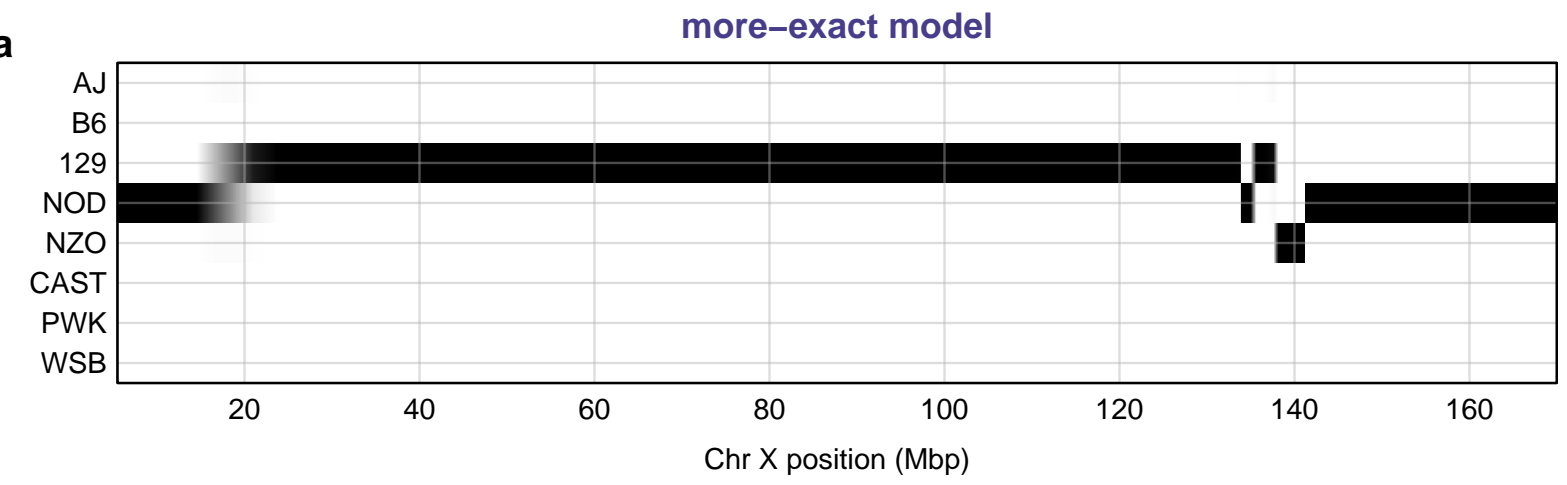

b

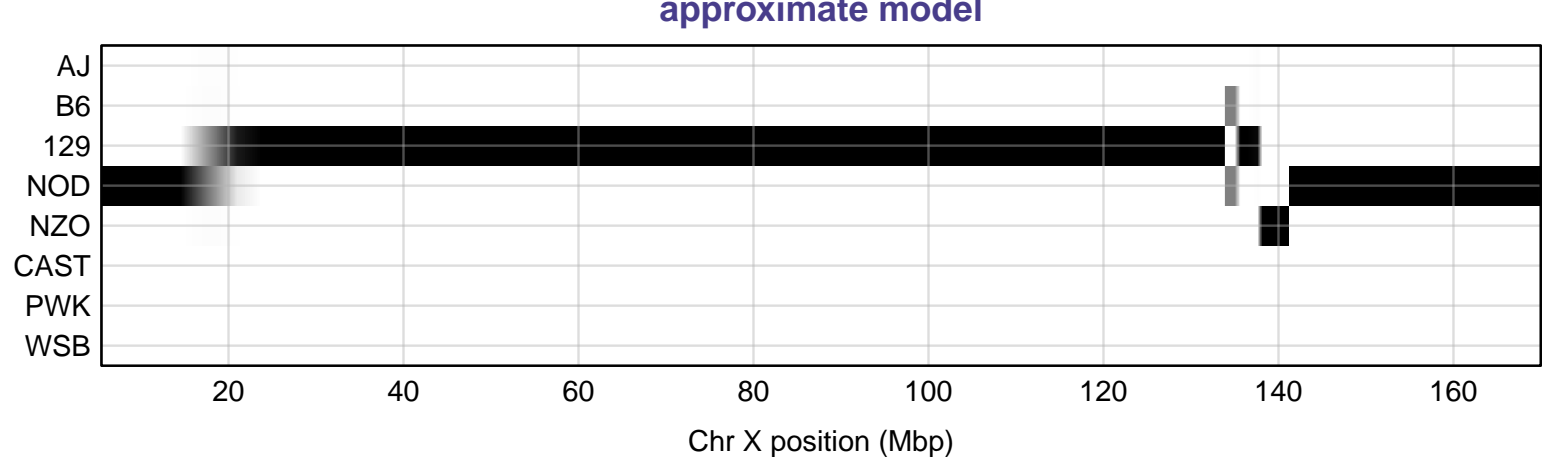

C

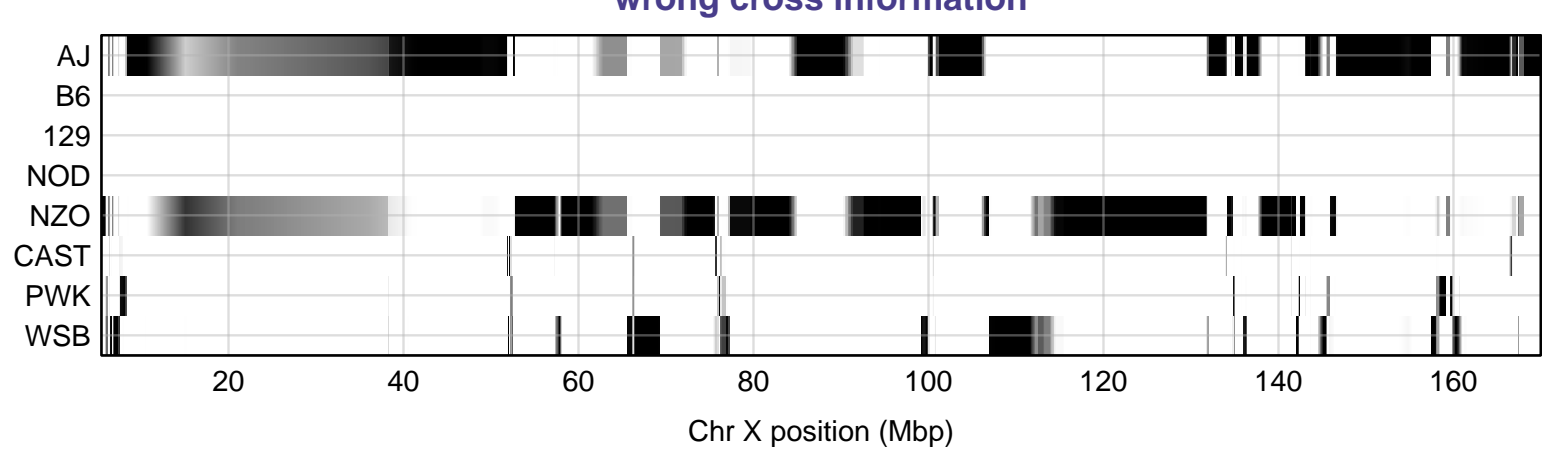

Figure 4 Genotype probabilities along the $\mathrm{X}$ chromosome for Collaborative Cross strain CC038/GeniUnc. a. Results using the more-exact model that excludes founders B6, CAST, and WSB. b. Results using the proposed approximate model. c. Results using the more-exact model but with the wrong cross information, excluding founders B6, 129, and NOD. 


\section{Discussion}

We have proposed an approximate model for use with genotype reconstruction in multi-parent populations (MPPs). We derived the two-point probabilities on autosomes in the case of random mating in large, discrete generations, derived from a founder population of a set of inbred lines in known proportions. We use the same frequencies for the $X$ chromosome, but with $2 / 3$ the number of generations. The approach is shown to give equivalent results for the mouse DO and CC populations, though with important differences for the $\mathrm{X}$ chromosome in $\mathrm{CC}$ lines, where some founder alleles can be excluded based on the cross design. The more-exact model for the $\mathrm{X}$ chromosome in the $\mathrm{CC}$ excludes three of the eight founders based on the cross design. This is particularly useful in cases that multiple founders are identical by descent across a region. However, the approximate model is not affected by errors in the specified cross design (see Figure 4).

The value of this generic model points towards the general usefulness of the original software for multi-parent populations, HAPPY (Mott et al. 2000), developed for the analysis of mouse heterogeneous stock. The results may depend on marker density and informativeness, but with a dense set of informative markers, a generic approach can provide good-quality genome reconstructions.

The hidden Markov model itself is an approximation. Meiosis generally exhibits positive crossover interference, but the Markov property is closer to being correct in multi-parent populations with multiple generations of mating, because nearby recombination events come from independent generations. This was apparent in the 3-point probabilities derived by Haldane and Waddington (1931) for two-way RIL and was further explored in Broman (2005) for 
multi-way RIL.

The proposed method has been implemented in the R/qt12 software (Broman et al. 2019a). It requires specification of the founder proportions and one other parameter (the number of generations of random mating) which governs the frequency of recombination breakpoints. The founder proportions should be straightforward from the cross design; the effective number of generations of random mating may require some calibration, such as through computer simulation to match the expected frequency of recombination breakpoints. 


\section{Data and software availability}

The R/qt12 software is available at the Comprehensive R Archive Network (CRAN), https: / / cran.r-project.org/package=qt12, as well as GitHub, https://github.com/rqt1/qt12. Further documentation is available at the R/qt12 website, https: //kbroman.org/qtl2.

The Diversity Outbred mouse data from Al-Barghouthi et al. (2021) is available at Zenodo, https://doi.org/10.5281/zenodo.4265417. Also see their companion repository of analysis scripts at GitHub, https://github.com/basel-maher/Do_project, and archived at Zenodo, https://doi .org/10.5281/zenodo. 4718146.

The Collaborative Cross mouse data from Srivastava et al. (2017) is available at Zenodo, https://doi.org/10.5281/zenodo.377036. Reorganized files in R/qt12 format are at https://github.com/rqtl/qtl2data/tree/master/CC.

Our detailed analysis code is available at GitHub, https://github.com/kbroman/Paper_GenericHMM, and archived at Zenodo, https://doi.org/10.5281/zenodo.5156660.

\section{Acknowledgments}

Two anonymous reviewers provided valuable comments for improvement of the manuscript.

\section{Funding}

This work was supported in part by National Institutes of Health grant R01GM070683. 
bioRxiv preprint doi: https://doi.org/10.1101/2021.08.03.454963; this version posted November 1, 2021. The copyright holder for this preprint (which was not certified by peer review) is the author/funder, who has granted bioRxiv a license to display the preprint in perpetuity. It is made available under aCC-BY 4.0 International license.

\section{Conflicts of interest}

The author declares that there is no conflict of interest. 


\section{Literature Cited}

Al-Barghouthi, B. M., L. D. Mesner, G. M. Calabrese, D. Brooks, S. M. Tommasini et al., 2021 Systems genetics in Diversity Outbred mice inform BMD GWAS and identify determinants of bone strength. Nat Commun 12: 1-19.

Bandillo, N., C. Raghavan, P. A. Muyco, M. A. L. Sevilla, I. T. Lobina et al., 2013 Multi-parent advanced generation inter-cross (MAGIC) populations in rice: progress and potential for genetics research and breeding. Rice 6: 1-15.

Broman, K. W., 2005 The genomes of recombinant inbred lines. Genetics 169: 1133-1146.

Broman, K. W., 2012a Genotype probabilities at intermediate generations in the construction of recombinant inbred lines. Genetics 190: 403-412.

Broman, K. W., 2012b Haplotype probabilities in advanced intercross populations. G3 (Bethesda) 2: 199-202.

Broman, K. W., D. M. Gatti, P. Simecek, N. A. Furlotte, P. Prins et al., 2019a R/qt12: software for mapping quantitative trait loci with high-dimensional data and multiparent populations. Genetics 211: 495-502.

Broman, K. W., D. M. Gatti, K. L. Svenson, Ś. Sen, and G. A. Churchill, 2019b Cleaning genotype data from Diversity Outbred mice. G3 (Bethesda) 9: 1571-1579.

Broman, K. W., and S. Sen, 2009 A guide to QTL mapping with R/qtl. Springer, New York.

Carter, T., and D. Falconer, 1951 Stocks for detecting linkage in the mouse, and the theory of their design. Journal of Genetics 50: 307-323. 
Cavanagh, C., M. Morell, I. Mackay, and W. Powell, 2008 From mutations to MAGIC: resources for gene discovery, validation and delivery in crop plants. Curr Opin Plant Biol 11: 215-221.

Churchill, G. A., D. M. Gatti, S. C. Munger, and K. L. Svenson, 2012 The diversity outbred mouse population. Mamm Genome 23: 713-718.

Complex Trait Consortium, 2004 The Collaborative Cross, a community resource for the genetic analysis of complex traits. Nat Genet 36: 1133-1137.

de Koning, D., and L. McIntyre, 2017 Back to the future: multiparent populations provide the key to unlocking the genetic basis of complex traits. Genetics 206: 527-529.

Dell'Acqua, M., D. M. Gatti, G. Pea, F. Cattonaro, F. Coppens et al., 2015 Genetic properties of the MAGIC maize population: a new platform for high definition QTL mapping in Zea mays. Genome Biol 16: 167.

Gatti, D. M., K. L. Svenson, A. Shabalin, L.-Y. Wu, W. Valdar et al., 2014 Quantitative trait locus mapping methods for diversity outbred mice. G3 (Bethesda) 4: 1623-1633.

Haldane, J. B. S., and C. H. Waddington, 1931 Inbreeding and linkage. Genetics 16: 357-374.

Huang, B. E., and A. W. George, 2011 R/mpmap: a computational platform for the genetic analysis of multiparent recombinant inbred lines. Bioinformatics 27: 727-729.

King, E. G., C. M. Merkes, C. L. McNeil, S. R. Hoofer, S. Sen et al., 2012 Genetic dissection of a model complex trait using the Drosophila Synthetic Population Resource. Genome Res 22: $1558-1566$. 
Kover, P. X., W. Valdar, J. Trakalo, N. Scarcelli, I. M. Ehrenreich et al., 2009 A multiparent advanced generation inter-cross to fine-map quantitative traits in Arabidopsis thaliana. PLoS Genet 5: e1000551.

Lincoln, S. E., and E. S. Lander, 1992 Systematic detection of errors in genetic linkage data. Genomics 14: 604-610.

Morgan, A. P., C.-P. Fu, C.-Y. Kao, C. E. Welsh, J. P. Didion et al., 2016 The Mouse Universal Genotyping Array: from substrains to subspecies. G3 (Bethesda) 6: 263-279.

Mott, R., C. J. Talbot, M. G. Turri, A. C. Collins, and J. Flint, 2000 A method for fine mapping quantitative trait loci in outbred animal stocks. Proc Natl Acad Sci USA 97: 12649-12654.

R Core Team, $2021 \mathrm{R}$ : A language and environment for statistical computing. R Foundation for Statistical Computing, Vienna, Austria.

Rabiner, L. R., 1989 A tutorial on hidden Markov models and selected applications in speech recognition. Proc. IEEE 77: 257-286.

Solberg Woods, L. C., C. Stelloh, K. R. Regner, T. Schwabe, J. Eisenhauer et al., 2010 Heterogeneous stock rats: a new model to study the genetics of renal phenotypes. Am J Physiol-Renal 298: F1484-F1491.

Srivastava, A., A. P. Morgan, M. L. Najarian, V. K. Sarsani, J. S. Sigmon et al., 2017 Genomes of the mouse Collaborative Cross. Genetics 206: 537-556.

Svenson, K. L., D. M. Gatti, W. Valdar, C. E. Welsh, R. Cheng et al., 2012 High-resolution genetic mapping using the mouse Diversity Outbred population. Genetics 190: 437-447. 
Teuscher, F., and K. W. Broman, 2007 Haplotype probabilities for multiple-strain recombinant inbred lines. Genetics 175: 1267-1274.

Yang, J., N. A. Zaitlen, M. E. Goddard, P. M. Visscher, and A. L. Price, 2014 Advantages and pitfalls in the application of mixed-model association methods. Nat Genet 46: 100-106.

Zheng, C., M. P. Boer, and F. A. van Eeuwijk, 2015 Reconstruction of genome ancestry blocks in multiparental populations. Genetics 200: 1073-1087.

Zheng, C., M. P. Boer, and F. A. van Eeuwijk, 2018 Accurate genotype imputation in multiparental populations from low-coverage sequence. Genetics 210: 71-82. 